\title{
INDEPENDENCIA EN USUARIOS DE SILLAS DE RUEDAS DURANTE SUS ACTIVIDADES DE LA VIDA DIARIA. UN ANÁLISIS USANDO LOS LENTES DE LA ERGONOMÍA Y EL DISEÑO
}

\author{
INDEPENDENCE IN WHEELCHAIR USERS THROUGHOUT DAILY LIFE ACTIVITIES. AN \\ APPROACH FROM THE PERSPECTIVE OF DESIGN AND ERGONOMICS
}

\author{
Pedro Castillo López ${ }^{1}$ \\ John Rey Galindo \\ Libertad Rizo Corona ${ }^{3}$ \\ Carlos Aceves González ${ }^{4}$
}

\begin{abstract}
Resumen: Las personas con discapacidad se enfrentan a la imposibilidad de llevar un estilo de vida independiente cuando se desenvuelven en entornos limitados. Para el análisis de esta problemática, la visión de la ergonomía resulta esencial, porque persigue la adecuación de los sistemas a las características, capacidades y limitaciones de los usuarios, para que puedan desarrollar sus actividades de la vida diaria con condiciones de igualdad, facilidad y seguridad. El presente trabajo explora el grado de independencia de usuarios de sillas de ruedas cuando realizan sus actividades de la vida diaria y su interacción con el entorno. Basado en un instrumento de evaluación de desempeño de las actividades de la vida diaria, se desarrolló y aplicó un cuestionario de 72 preguntas distribuidas en 10 secciones. Se identificaron las actividades con menor independencia en usuarios permanentes de silla de ruedas y su relación con las propiedades ergonómicas del entorno y la experiencia de uso. Diez usuarios de silla de ruedas participaron en el estudio. Se encontró que las actividades con mayor dependencia y conflictos de interacción fueron cocinar, lavar la ropa, realizar compras y usar el transporte público; las actividades básicas y de autocuidado como bañarse, vestirse, utilizar el baño y el desplazamiento personal fueron consideradas con cierta independencia. Con este trabajo se aspira a contribuir a la comprensión de la necesidad de mejorar la calidad de vida de las personas con discapacidad y de visibilizar la importancia de la ergonomía y el diseño para lograrlo.
\end{abstract}

Palabras clave: Ergonomía, discapacidad, independencia.

Abstract: People with disabilities confront the impossibility of living an independent lifestyle when they perform in limited environments. For the analysis of this problem, the vision of ergonomics is essential, because it pursues the adaptation of systems to the characteristics, capabilities, and limitations of users so that they can develop their daily activities with equality, simplicity, and safety.

${ }^{1}$ Universidad de Guadalajara. Guadalajara, México. Correo electrónico: diperickcalo@gmail.com. Orcid: https://orcid.org/o0oo-0002-8233-2804

${ }^{2}$ Universidad de Guadalajara. Guadalajara, México. Correo electrónico: john.rey@academicos.udg.mx. Orcid: https://orcid.org/oooo-0002-0458-7107

3Universidad de Guadalajara. Guadalajara, México. Correo electrónico: libertad.rizo.din@gmail.com. Orcid: https://orcid.org/oooo-0001-5288-1610

4Universidad de Guadalajara. Guadalajara, México. Correo electrónico: c.aceves@academicos.udg.mx. Orcid: https://orcid.org/oooo-0002-6720-808X 
This work explores the degree of independence of wheelchair users when performing their daily life activities and their interaction with the environment. Based on a performance evaluation instrument of the activities of daily living, a questionnaire of 72 questions distributed in 10 sections was developed and applied. The activities with less independence in permanent wheelchair users were identified and their relationship with the ergonomic properties of the environment and the experience of use. Ten wheelchair users participated in the study. The activities with the highest dependence and interaction conflicts were found to be cooking, laundry, shopping, and using public transportation; basic and selfcare activities such as bathing, dressing, using the bathroom, and personal movement was considered to have some independence. This work aims to contribute to the understanding of the need to improve the quality of life of people with disabilities and to make visible the importance of ergonomics and design to this end.

Keywords: Ergonomics, disability, independency.

Recepción: 05.11.2020 / Revisión: 12.11.2020 / Aceptación: 03.12.2020

\section{Introducción}

Vivir en condición de discapacidad implica un impacto negativo en los derechos fundamentales y el acceso al bienestar en igualdad de oportunidades. Esto a partir de la afectación de la libertad para desarrollar una vida en total independencia (Organización de las Naciones Unidas [ONU], 2014).

La independencia personal es un derecho fundamental establecido en el artículo 19 de la Convención sobre los derechos de las personas con discapacidad (CDPD) (ONU, 2014), que se define como la capacidad del individuo para satisfacer sus necesidades básicas, o realizar las actividades de la vida diaria (Romero-Ayuso, 2007).

Las actividades básicas de la vida diaria (ABVD) describen aquellas actividades que resultan esenciales y que se realizan diaria, habitual y universalmente, como alimentarse, vestirse o usar el baño. En el caso de las actividades instrumentales de la vida diaria (AIVD), estas se diferencian de las anteriores, por requerir organización y planeación (Johnson et al., 2004), además del uso de utensilios, como en el caso de cocinar, hacer compras o llamar por teléfono.

Tanto las ABVD como las AIVD resultan importantes para estudiar la reducción de capacidades y autocuidado (Hartigan, 2007; Hilgenkamp et al., 2011) y actúan como referencia de la autonomía e independencia de las personas en la vida cotidiana. Sin condiciones adecuadas, las personas con discapacidad requieren en muchos casos de la asistencia de otros para realizar las ABDV y las AIVD; e incluso para la toma de decisiones que, bajo otras circunstancias, serían su plena responsabilidad. Este fenómeno genera escenarios que vulneran la privacidad y autodeterminación, lo que repercute en una baja calidad de vida y satisfacción.

En México, según datos de la Encuesta Nacional de la Dinámica Demográfica (ENADID) (INEGI, 2018), el 52\% de la población con discapacidad en el país está constituida por aquella que tiene dificultad para caminar, subir o bajar escaleras usando sus piernas. 
Muchos de estos usuarios requieren de una silla de ruedas para garantizar su movilidad. Sin embargo, la garantía sobre la movilidad no implica una vida independiente en la realización de todas las ABVD y las AIVD y con ello en el bienestar.

La Clasificación Internacional del Funcionamiento de la Discapacidad y la Salud define la discapacidad como el resultado de una interacción entre la(s) condición(es) de salud de una persona y el entorno físico, humano, actitudinal y sociopolítico en el que vive la persona (OMS [Organización Mundial de la Salud], 2001). Esta definición planteada desde el modelo social y no médico, evidencia que la discapacidad es el resultado de la interacción entre las desproporcionadas demandas del entorno frente a las capacidades del usuario. Bajo esta idea, la discapacidad y con ella la independencia, deben ser estudiadas desde una perspectiva sistémica. Esto con el fin de comprender la relación entre los componentes e identificar los elementos determinantes para el surgimiento de estos fenómenos.

Es esencial para comprender con mayor claridad mirar el fenómeno de la dependencia, considerando la experiencia de los individuos y el papel del entorno y los objetos a partir de sus características, en su carácter de posibilitadores o limitadores (Persad et al., 2007). Esto permite reconocer las áreas de oportunidad para mejorar el desempeño, eliminar los esfuerzos innecesarios, facilitar la realización de las tareas y evitar la exposición al riesgo de accidentes o lesiones.

El presente documento tiene como propósito evidenciar los grados de independencia de usuarios permanentes de sillas de ruedas en la realización de sus ABVD y AIVD y el papel que tienen aspectos como el entorno y los objetos con relación a la independencia y el bienestar. Para lo anterior, se consideran las actividades en las que los usuarios identifican una menor independencia y las razones asociadas Además de esto, se indaga sobre la experiencia del usuario con relación a la silla de ruedas, siendo este un aspecto básico en la realización de las actividades cotidianas.

\section{Materiales y métodos}

\section{Participantes}

En el estudio participaron cinco mujeres y cinco hombres con un promedio de edad de 33 años y un mínimo de cinco años de experiencia en el uso de la silla de ruedas, de manera permanente.

\section{Instrumentos}

Utilizando la herramienta de evaluación de desempeño de las actividades propuesto por Neumann-Collyer et al. (2018) se construyó un cuestionario compuesto por 10 secciones y 72 preguntas en total, agrupadas por actividad y variable. Las preguntas fueron de elección múltiple, teniendo entre tres y cinco opciones posibles de respuesta.

El cuestionario fue enviado y respondido en línea y como información previa se les comunicó a los participantes el tiempo aproximado necesario para responder ( 25 minutos) y 
se les pidió su consentimiento para participar en el estudio.

El cuestionario está enfocado en el nivel de desempeño y se compone por razones de por qué no realiza la actividad, dependencia y el nivel de importancia del desempeño de dicha actividad; dimensiones propuestas por Neumann-Collyer et al., (2018). A estas tres dimensiones se sumó una cuarta dimensión que indaga sobre la percepción que tienen los participantes de los entornos donde realizan las actividades y su rol frente a la realización de sus actividades. Se analizaron como ABVD, bañarse, vestirse, usar el baño y desplazarse y como AIVD, lavar ropa, preparar alimentos, limpiar la cocina, ir de compras, usar el transporte, realizar rehabilitación, realizar actividad física y socializar. Estas variables, asi como sus indicadores observables se describen a continuación en la tabla 1.

Tabla 1. Variables sobre independencia.

\begin{tabular}{|c|c|c|}
\hline $\begin{array}{l}\text { Constructo } \\
\text { conceptual }\end{array}$ & $\begin{array}{c}\text { Variable } \\
\text { operacional }\end{array}$ & Indicador observable \\
\hline \multirow{18}{*}{ 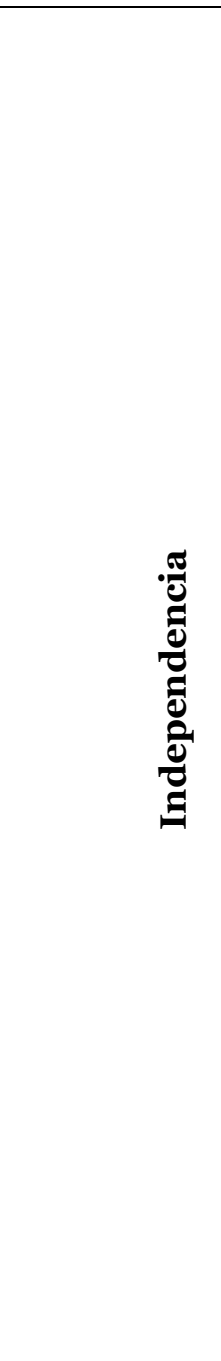 } & \multirow{4}{*}{$\begin{array}{l}\text { Nivel de } \\
\text { desempeño }\end{array}$} & $\begin{array}{l}\text { No lo hago, alguien más realiza la actividad por } \\
\text { mi. }\end{array}$ \\
\hline & & $\begin{array}{l}\text { Participo en la actividad ejecutando ciertas } \\
\text { operaciones (realizo menos del } 75 \% \text { ). }\end{array}$ \\
\hline & & $\begin{array}{l}\text { Lo hago con vigilancia, apoyo o muestra de } \\
\text { tercero (apoyo físico mínimo). }\end{array}$ \\
\hline & & $\begin{array}{l}\text { Lo hago sin asistencia de un tercero, aunque } \\
\text { utilice ayudas técnicas, ortesis u otros. }\end{array}$ \\
\hline & \multirow{6}{*}{$\begin{array}{l}\text { Razón de } \\
\text { dependencia }\end{array}$} & Mi discapacidad me lo impide. \\
\hline & & Por razones de género o tradición. \\
\hline & & $\begin{array}{l}\text { No tengo el dinero para hacerlo o comprar lo } \\
\text { necesario. }\end{array}$ \\
\hline & & $\begin{array}{l}\text { El espacio no está adaptado para mi y/o mi silla } \\
\text { de ruedas no me lo permite. }\end{array}$ \\
\hline & & $\begin{array}{l}\text { Tuve un accidente antes y tengo miedo al realizar } \\
\text { la actividad. }\end{array}$ \\
\hline & & No me gusta hacerlo. \\
\hline & \multirow{4}{*}{$\begin{array}{l}\text { Condiciones del } \\
\text { entorno }\end{array}$} & $\begin{array}{l}\text { Óptimas para realizar la actividad sin ayuda o } \\
\text { supervisión. }\end{array}$ \\
\hline & & $\begin{array}{l}\text { Permiten realizar la actividad, pero con apoyo o } \\
\text { dificultad. }\end{array}$ \\
\hline & & No permiten realizar la actividad. \\
\hline & & $\begin{array}{l}\text { No permiten realizar la actividad y me ponen en } \\
\text { peligro. }\end{array}$ \\
\hline & \multirow{4}{*}{$\begin{array}{l}\text { Importancia de la } \\
\text { actividad }\end{array}$} & No importante. \\
\hline & & Indistinto. \\
\hline & & Importante. \\
\hline & & Muy importante. \\
\hline
\end{tabular}

Nota. Se utiliza como referencia el instrumento para la evaluación de las actividades de la vida diaria de Neumann-Collyer et al. (2018). 
Una segunda parte del cuestionario se enfocó en conocer la experiencia de uso sobre la silla de ruedas a través de la percepción que tienen de su apariencia y lo que perciben de sí mismos usándola, su funcionalidad y seguridad y finalmente lo que consideran que perciben los otros de ellos usando la silla. Lo anterior está basado en los planteamientos de la experiencia del usuario en los niveles visceral, conductual y reflexivo de (Norman, 2005). En la tabla 2 se muestran las variables operacionales sobre la experiencia de uso, sí cómo los indicadores observables correspondientes.

Tabla 2. Variables sobre experiencia del usuario.

\begin{tabular}{|c|c|c|}
\hline $\begin{array}{l}\text { Constructo } \\
\text { conceptual }\end{array}$ & Variable operacional & Indicador observable \\
\hline \multirow{8}{*}{ 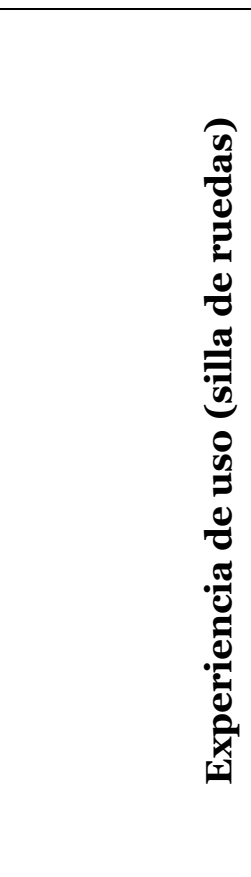 } & \multirow{2}{*}{$\begin{array}{l}\text { Percepción sobre la apariencia } \\
\text { del producto }\end{array}$} & $\begin{array}{l}\text { Me agrada su apariencia y me } \\
\text { gusta cómo me veo usándola. }\end{array}$ \\
\hline & & $\begin{array}{l}\text { No me agrada como se ve y no } \\
\text { me gusta cómo me veo yo } \\
\text { usándola. }\end{array}$ \\
\hline & \multirow{4}{*}{$\begin{array}{l}\text { Percepción de la funcionalidad } \\
\text { del producto }\end{array}$} & $\begin{array}{l}\text { Todo funciona correctamente y } \\
\text { me permite desarrollar la } \\
\text { actividad con facilidad. }\end{array}$ \\
\hline & & $\begin{array}{l}\text { Todas sus partes funcionan bien, } \\
\text { pero su forma o dimensiones } \\
\text { complican realizar la actividad. }\end{array}$ \\
\hline & & $\begin{array}{l}\text { Algunas partes no funcionan bien } \\
\text { y se descompone con frecuencia. }\end{array}$ \\
\hline & & $\begin{array}{l}\text { En ocasiones he sufrido } \\
\text { accidentes o caídas por } \\
\text { deficiencias funcionales de mi } \\
\text { silla. }\end{array}$ \\
\hline & \multirow{2}{*}{$\begin{array}{l}\text { Percepción sobre proyección } \\
\text { social* }^{*}\end{array}$} & $\begin{array}{l}\text { Me gusta cómo me miran los } \\
\text { demás por la silla que uso. }\end{array}$ \\
\hline & & $\begin{array}{l}\text { No me gusta lo que la gente dice } \\
\text { de mi por la silla que uso. }\end{array}$ \\
\hline
\end{tabular}

Nota. La variable sobre proyección social tiene relación sobre cómo se percibe el usuario frente a los demás mientras usa la silla de ruedas, como lo miran y la imagen que considera que transmite.

\section{Materiales y equipo}

El cuestionario fue elaborado y aplicado a través de la plataforma Google Forms. El acceso fue controlado por el investigador, los participantes requirieron del uso de un dispositivo móvil o computadora para el acceso al cuestionario. 


\section{Resultados}

\section{Actividades con independencia}

Las respuestas de los participantes que refieren el nivel de desempeño con el que realizan las ABVD (bañarse, vestirse, usar el baño y desplazarse) en ocho de los diez participantes se realizan de forma independiente sin la asistencia de alguien más. Para ello, reportan el uso de apoyos físicos y de elementos auxiliares en la realización de la actividad, como sillas, soportes, etc.

Con respecto a la razón de dependencia, en dos de los diez participantes se menciona que se encuentra relacionada con el entorno y las condiciones no adaptadas, uno con el tipo de discapacidad y los siete participantes restantes no respondieron porque se consideran a sí mismos totalmente independientes durante estas actividades.

\section{Actividades con dependencia}

De acuerdo con los objetivos propuestos se presentan las actividades con mayor dependencia en la tabla 3, en donde al menos la mitad de los participantes realizan dichas actividades con dependencia y asistencia o ayuda de alguien más; estas actividades están relacionadas con el entorno como razón de dependencia en siete de diez participantes para las actividades de lavar la ropa, preparar alimentos y limpiar la cocina; el entorno afecta de la misma manera al realizar las compras, utilizar el transporte y recibir rehabilitación en al menos tres de los diez participantes en el estudio.

En las actividades descritas con alta dependencia por los participantes, se menciona también que las condiciones del entorno se encuentran adaptadas apenas en tres de diez casos para realizar la actividad según las necesidades particulares del usuario. Puede verse en detalle en la tabla 3, que todas las actividades son reportadas como importantes o muy importantes para la mayoría de los participantes. 
Tabla 3. Resumen de actividades con dependencia.

\begin{tabular}{|c|c|c|c|c|c|}
\hline & & $\begin{array}{c}\text { Nivel de } \\
\text { desempeño }\end{array}$ & $\begin{array}{c}\text { Razón de } \\
\text { dependenci } \\
\text { a }\end{array}$ & $\begin{array}{c}\text { Condicione } \\
\text { s del } \\
\text { entorno } \\
\end{array}$ & Importancia \\
\hline & Actividad & $\begin{array}{l}\text { Participantes } \\
\text { con } \\
\text { independenci } \\
\text { a durante la } \\
\text { actividad }\end{array}$ & $\begin{array}{c}\text { Participantes } \\
\text { que } \\
\text { mencionan } \\
\text { que la } \\
\text { dependencia } \\
\text { es por el } \\
\text { entorno }\end{array}$ & $\begin{array}{c}\text { Participantes } \\
\text { con } \\
\text { condiciones } \\
\text { adaptadas en } \\
\text { el entorno }\end{array}$ & $\begin{array}{c}\text { Actividad } \\
\text { importante y } \\
\text { muy } \\
\text { importante }\end{array}$ \\
\hline \multirow{4}{*}{ 召 } & Bañarse & $8 / 10$ & $2 / 10$ & $4 / 10$ & $10 / 10$ \\
\hline & Vestirse & $8 / 10$ & $2 / 10$ & $6 / 10$ & $10 / 10$ \\
\hline & Usar el baño & $9 / 10$ & $2 / 10$ & $4 / 10$ & $10 / 10$ \\
\hline & Desplazamiento & $8 / 10$ & $2 / 10$ & $4 / 10$ & $10 / 10$ \\
\hline \multirow{8}{*}{$\sum$} & Lavar ropa & $3 / 10$ & $7 / 10$ & $3 / 10$ & $10 / 10$ \\
\hline & Preparar alimentos & $4 / 10$ & $7 / 10$ & $3 / 10$ & $9 / 10$ \\
\hline & Limpiar Cocina & $4 / 10$ & $7 / 10$ & $3 / 10$ & $9 / 10$ \\
\hline & Compras & $5 / 10$ & $4 / 10$ & $3 / 10$ & $10 / 10$ \\
\hline & Transporte & $5 / 10$ & $5 / 10$ & $3 / 10$ & $10 / 10$ \\
\hline & Rehabilitación & $5 / 10$ & $3 / 10$ & $4 / 10$ & $10 / 10$ \\
\hline & Actividad Física & $7 / 10$ & $1 / 10$ & $7 / 10$ & $10 / 10$ \\
\hline & Socializar & $8 / 10$ & $3 / 10$ & $3 / 10$ & $10 / 10$ \\
\hline
\end{tabular}

Nota. Los resultados se presentan en referencia al número de participantes que dieron la respuesta que se muestra.

\section{El uso del transporte}

Con el interés de conocer de forma general el tipo de desplazamientos con movilidad independiente de los usuarios y la interacción que acostumbran con el entorno fuera de su casa, se hicieron cuestionamientos sobre el tipo de transporte que normalmente utilizan y los lugares que se frecuentan para socializar con otras personas.

Cuando se preguntó sobre el tipo de transporte utilizado para desplazarse de un lugar a otro se registró más de una respuesta por participante debido a la naturaleza de sus actividades; sobre esto, ocho participantes (45\%) mencionaron utilizar con normalidad un automóvil propio, de un familiar o un amigo y, de forma extraordinaria, cuatro participantes $(22,2 \%)$ utilizan servicio de taxi o aplicaciones para adquirir un servicio semejante. La frecuencia de uso del transporte público, como el camión urbano, lo reportan tres participantes (17\%) y, de igual forma, tres participantes (17\%) reportan rodar por las calles con el impulso de sus brazos.

El total de desplazamientos diarios se agruparon en 3 categorías de acuerdo a proporciones de tiempo; seis de diez participantes respondieron que un $25 \%$ de su tiempo del día se desplazan utilizando su silla de ruedas utilizando el impulso de sus brazos y el $75 \%$ de su tiempo utilizan otro tipo de transporte; tres de diez participantes distribuyen su dinámica cotidiana entre 50\% de uso de un transporte y 50\% en su silla de ruedas con el impulso de sus brazos; finalmente, uno de ellos respondió que utiliza en un $75 \%$ el impulso de sus brazos en combinación con el uso del transporte en un $25 \%$ de sus desplazamientos diarios. 


\section{Sobre la interacción social}

Las actividades que más realizan los participantes para socializar y relacionarse con otros son: salir al cine (18\%), trabajar (17\%), salir a comer (17\%), y pasear en lugares públicos durante el día (16\%); en menor medida los participantes acuden a conciertos (12\%), estudian (6\%) y salen al parque (2\%).

El nivel de relevancia en relación con estas actividades es reportado como muy importante y las condiciones que se consideran deseables para desenvolverse jerárquicamente son: que el lugar cuente con baños adaptados (nueve participantes); que el lugar tenga accesibilidad física como rampas, elevadores, etc. (ocho participantes); y que el lugar le permita desenvolverse en total independencia (seis participantes). Al mismo tiempo, aunque con menor estima, se menciona la atención de las personas que ahí se encuentran (cinco participantes).

\section{Experiencia del usuario}

Sobre la silla de ruedas que utilizan, siete participantes mencionan utilizar un modelo independiente, dos utilizan una silla de ruedas hospitalaria y uno de ellos hace uso de una silla de ruedas eléctrica. La silla de ruedas de modelo independiente, se caracteriza por utilizar respaldo sin mangos de empuje para asistencia, tener una estructura rígida y el reposapiés cercano a las ruedas delanteras; algunos modelos permiten el plegado del respaldo en un sentido vertical. La silla de ruedas hospitalaria es plegable en sentido horizontal con reposapiés independientes de la estructura, asiento y respaldo de textil tensado y apoya brazos laterales, inventada por Harry Jennings en 1932. La silla de ruedas eléctrica, se distingue de otras sillas de ruedas por su mecanismo de desplazamiento mecánico y accionamiento por control que se encuentra sobre uno de los descansa brazos, normalmente no se pliegan más allá del respaldo, sus dimensiones son mayores que otras tipologías.

De acuerdo a los participantes, la forma en que adquirieron la silla de ruedas fue por donación en nueve de los casos, en donde fueron tomadas medidas antropométricas y se indagó sobre las características físicas del usuario (peso, talla, edad, estilo de vida, etc.); sin embargo, no eligieron aspectos como materiales, forma y apariencia.

Sobre las lesiones o complicaciones de salud, agrupadas dentro del 68\% de las respuestas de los participantes se mencionan, por su frecuencia de ocurrencia, caídas y golpes por deficiencias del objeto o el espacio; dolor de espalda, y deficiencia circulatoria en miembros inferiores (hinchazón). Por otra parte, con menor frecuencia en 32\%, se reportan lesiones (ampollas) en dedos o palmas de la mano; lesiones en hombros y brazos, y ulceras por presión en glúteos o zonas cercanas.

Cuando se les preguntó sobre la percepción de la silla de ruedas (nivel visceral), la respuesta de ocho participantes fue que les agrada de forma general su apariencia y les gusta la imagen que proyectan al usarla. En lo relacionado con su funcionamiento (nivel conductual), las respuestas de cinco participantes fue que se cumplen al máximo sus expectativas, todo funciona bien y les permite realizar sus actividades sin ayuda; por último, en lo correspondiente a las reacciones de otras personas frente al uso de su silla de ruedas en 
interacción social, seis participantes respondieron que les gusta cómo los ven los demás, lo que piensan y dicen por el tipo de silla que utilizan. En la tabla 4 se muestra un resumen sobre la experiencia del usuario, en donde se señalan las variables operacionales, el indicador observable y su correspondencia de acuerdo con el tipo de silla de ruedas que utiliza.

Tabla 4. Resumen de experiencia del usuario.

\begin{tabular}{|c|c|c|c|}
\hline $\begin{array}{l}\text { Variable } \\
\text { operacional }\end{array}$ & Indicador observable & $\begin{array}{l}\text { Tipo de silla } \\
\text { usada* }\end{array}$ & $\begin{array}{l}\text { Número de } \\
\text { participantes }\end{array}$ \\
\hline \multirow{2}{*}{$\begin{array}{l}\text { Percepción } \\
\text { sobre la } \\
\text { apariencia del } \\
\text { producto }\end{array}$} & $\begin{array}{l}\text { Me agrada su apariencia y me } \\
\text { gusta cómo me veo usándola }\end{array}$ & $\begin{array}{l}\text { Independiente } \\
\text { Hospitalaria }\end{array}$ & $\begin{array}{l}7 / 7 \\
1 / 2\end{array}$ \\
\hline & $\begin{array}{l}\text { No me agrada como se ve y no } \\
\text { me gusta cómo me veo yo } \\
\text { usándola }\end{array}$ & $\begin{array}{l}\text { Hospitalaria } \\
\text { Eléctrica }\end{array}$ & $\begin{array}{l}1 / 2 \\
1 / 1\end{array}$ \\
\hline \multirow{3}{*}{$\begin{array}{l}\text { Percepción de } \\
\text { la } \\
\text { funcionalidad } \\
\text { del producto }\end{array}$} & $\begin{array}{l}\text { Todo funciona correctamente y } \\
\text { me permite desarrollar la } \\
\text { actividad con facilidad }\end{array}$ & $\begin{array}{c}\text { Independiente } \\
\text { Hospitalaria }\end{array}$ & $\begin{array}{l}4 / 7 \\
1 / 2\end{array}$ \\
\hline & $\begin{array}{l}\text { Todas sus partes funcionan } \\
\text { bien, pero su forma o } \\
\text { dimensiones complican realizar } \\
\text { la actividad }\end{array}$ & $\begin{array}{l}\text { Independiente } \\
\text { Hospitalaria } \\
\text { Eléctrica }\end{array}$ & $\begin{array}{l}2 / 7 \\
1 / 2 \\
1 / 1\end{array}$ \\
\hline & $\begin{array}{l}\text { Algunas partes no funcionan } \\
\text { bien y se descompone con } \\
\text { frecuencia }\end{array}$ & Independiente & $1 / 7$ \\
\hline \multirow{2}{*}{$\begin{array}{l}\text { Percepción } \\
\text { sobre } \\
\text { proyección } \\
\text { social }\end{array}$} & $\begin{array}{l}\text { Me gusta cómo me miran los } \\
\text { demás por la silla que uso }\end{array}$ & $\begin{array}{c}\text { Independiente } \\
\text { Hospitalaria }\end{array}$ & $\begin{array}{l}7 / 7 \\
1 / 2\end{array}$ \\
\hline & $\begin{array}{l}\text { No me gusta lo que la gente dice } \\
\text { de mi por la silla que uso }\end{array}$ & $\begin{array}{c}\text { Eléctrica } \\
\text { Hospitalaria }\end{array}$ & $\begin{array}{l}1 / 1 \\
1 / 2\end{array}$ \\
\hline
\end{tabular}

Nota. Dentro de cada tipología de sillas de ruedas existen variaciones entre modelos que pueden ofrecer distintas características para los usuarios, sin embargo, se clasifican en dichas categorías por los mecanismos de sus partes principales (ruedas, respaldo, asiento y reposapiés).

\section{Discusión}

Según los resultados de este estudio, se observó que principalmente durante las ABVD existe mayor nivel de independencia. Los resultados señalan que la independencia lograda tiene una relación con ajustes al entorno que les permite desarrollar con mayor libertad y autonomía sus actividades básicas. Según el Instituto Nacional de Salud Pública (2013) un $77,8 \%$ de los hogares en donde vive una persona con problemas motrices reportó la necesidad de modificar el interior de la vivienda en espacios como el baño, las escaleras y la recamara del usuario para favorecer su autonomía.

Si bien en las ABVD se reconoce un mayor número de personas con independencia, resulta interesante que esto disminuye cuando se habla de AIVD. Esta situación coincide con lo reportado en estudios con otras poblaciones como la de adultos mayores (Van-Rensbergen \& Pacolet, 2012; Ran et al., 2017; Arenas-Jiménez et al., 2019) donde también se ha reconocido que hay menor independencia cuando se trata de AIVD en comparación con las 
ABVD. Una probable razón es que las AIVD son actividades con un más alto nivel de complejidad (Mlinac y Feng, 2016). En este sentido, surge la necesidad de reconocer qué aspectos de la actividad y de los recursos que se usan para su realización deben ser rediseñados.

Debido a que la independencia para desarrollar actividades relacionadas con la intimidad es fundamental para la calidad de vida de las personas (Lysack et al., 2001), las personas hacen un esfuerzo mayor para lograr desarrollarlas sin ayuda. Esto sugiere que el usuario se preocupa por mantener condiciones óptimas tanto en su relación con el entorno y los recursos físicos que requiere para estos fines.

En relación con las AIVD, quehaceres domésticos como lavar ropa, limpiar o cocinar, se reportan como actividades en las que existe un menor nivel de independencia. Para el caso de estas tres actividades, la falta de adecuación del entorno se reporta como la razón principal de la dependencia. Esto confirma la necesidad de seguir desarrollando investigación en esta área. Resulta relevante para el diseño de productos, servicios y sistemas que resuelvan estas problemáticas y para ello se requiere de la información y estructura teórica que aporta la ergonomía.

Si bien la independencia en las ABVD y en las AIVD generalmente se trata por separado (Spector y Fleishman, 1998; Millán-Calenti et al. 2010), se ha identificado también que el sumar lo que sucede con las AIVD a las ABVD, como descriptores de la discapacidad, puede dar un panorama multidimensional que permitiría entender de mejor manera cómo se relacionan las múltiples variables que generan situaciones de discapacidad (Spector \& Fleishman, 1998). En este sentido, el analizar detalladamente las condiciones que se presentan en la realización de las AIVD con mayor reporte de dependencia, podría dar mayor claridad sobre el escenario de la discapacidad.

Las respuestas de los usuarios sobre socialización o esparcimiento dan cuenta de un estilo de vida con tendencia hacia la convivencia y participación social. Según su percepción, la socialización es un factor muy importante en su vida diaria. Esta información resulta relevante considerando la correlación positiva que se ha encontrado entre la socialización y la calidad de vida relacionada con la salud (Sun et al., 2011). Sin embargo, es importante considerar las razones por las cuales las actividades de esparcimiento y socialización se pueden ver afectadas. Algunos estudios sugieren que hasta el 60,5\% de la población con discapacidad, ha encontrado dificultades en esta actividad, considerando la discapacidad misma $(36,6 \%)$ como un factor negativo para ello y mencionando algunos otros como el transporte (Instituto Nacional de Salud Pública, 2013). En cuanto a las condiciones que reconocen como esenciales en los lugares para socializar, se mencionan tanto baños bien adaptados como aspectos de accesibilidad física.

Respecto a la experiencia de uso de la silla de ruedas, resulta relevante señalar que si bien, en nueve de los casos se señala una adecuación de carácter antropométrico en la silla, también se hace referencia sobre dolencias en espalda y problemas relacionados con la circulación y la piel. En este sentido, se requiere de un análisis más claro de las características de las sillas, con el fin de ofrecer mejores alternativas a los problemas de diseño que está presentando (Gayol-Mérida et al., 2014). 
También, se reporta una relativa frecuencia de caídas o golpes que generan lesiones y complicaciones de salud, dichas respuestas pueden compararse con los resultados presentados por Chen et al. (2011), en donde se encontró una prevalencia de un accidente en el $54,7 \%$ de los participantes y un $16,8 \%$ que reportó dos o más episodios de accidentes durante el período de 3 años. Lo que puede estar relacionado con el esfuerzo por parte del usuario para realizar cualquier actividad a pesar de las barreras que puede representar un entorno no adaptado correctamente en espacios reducidos, sin rampas o elevadores, poniéndose en riesgo.

En cuanto a lo reportado con relación a la percepción de la apariencia de la silla, su funcionalidad y su proyección social, llama la atención que la mayoría están conformes con la apariencia de la silla que usan. Sin embargo, aparecen resultados menos positivos cuando se trata de la funcionalidad y la proyección social. En el caso de la funcionalidad, tan solo el 50\% tiene una buena percepción y los demás reportan problemas de practicidad que se relacionan directamente con la independencia; lo que refuerza lo mencionado anteriormente en relación con la necesidad del rediseño de la silla. En el caso de la proyección social, el 40\% no percibe positivamente lo que piensan los demás de ellos por el tipo de silla de ruedas que utilizan, lo que constituye un llamado a reconocer factores de carácter social por parte de quienes determinan las características del objeto.

La satisfacción completa del usuario sobre todas las partes de su silla de ruedas es fundamental. Aun cuando se tiene acceso a ella por donación, es importante tener en cuenta la experiencia del usuario para configurar cada parte según las necesidades. No cualquier silla es mejor que nada, como reporta Visagie et al. (2015), en donde más del 60\% de los participantes se mostraron insatisfechos con el uso de una silla de ruedas con adaptaciones parecidas al de tipo hospitalaria, sobre las características de durabilidad, fiabilidad, facilidad de ajuste, eficacia y seguridad.

Los resultados de este estudio ofrecen un acercamiento sobre los niveles de independencia de los usuarios permanentes de sillas de ruedas al realizar sus actividades de la vida diaria. No obstante, también es cierto que el estudio presenta algunas limitaciones. Quizá la mayor limitación está relacionada con el número de participantes. Sin embargo, esa misma limitación sugiere la importancia de realizar investigación con una muestra más amplia y también incrementar la diversidad de usuarios, teniendo una mayor participación de usuarios con diversos perfiles socioeconómicos.

En el mismo sentido, es esencial analizar lo que sucede con personas con menos experiencia en el desarrollo de una nueva vida en condición de discapacidad, involucrando etapas de adaptación o curva de aprendizaje que pueden diferir de la experiencia de uso de los participantes en este estudio. Es importante también considerar a aquellos usuarios no permanentes de silla de ruedas que hacen uso de este elemento por periodos intermitentes o limitados a un periodo particular, quienes probablemente puedan presentar requerimientos, experiencias y percepciones diferentes. 


\section{Conclusiones}

Existen soluciones particulares al problema de la discapacidad que pueden dar respuesta momentánea a la necesidad de independencia. Sin embargo, sigue siendo releavante desarrollar soluciones desde una perspectiva sistémica que considere todos los elementos que interactúan para generar la discapacidad. Con ello, se pueden incrementar las probabilidades de satisfacer las necesidades de los usuarios en interacción con el contexto. Por ejemplo, es posible que una silla de ruedas sea cómoda y segura, pero que sus dimensiones definidas por la búsqueda de estos dos objetivos no permitan que pase por la puerta de cualquier habitación, o en la audacia de diseñar la silla más ligera y económica, esta resulte incomoda y hasta peligrosa. La realidad ideal para cada usuario es aquella que está cercana a sus necesidades individuales; cada silla de ruedas debe permitir ajustarse de manera particular a la necesidad para quien la usa.

Posiblemente, al analizar cada una de las actividades reportadas como de alta dependencia, se pueda dilucidar qué tipo de objetos o espacios no son compatibles con la independencia de las personas con discapacidad motriz, y de qué forma podrían ser mejorados para garantizar que todos los usuarios puedan acceder en igualdad de oportunidades. Es esencial buscar escenarios de mejora de la experiencia de uso para todos, y contribuir a la mejora de la calidad de vida desde soluciones de diseño enriquecidas y previstas por una perspectiva ergonómica e incluyente.

\section{Referencias}

Arenas-Jiménez, M. D., Navarro-García, M., Serrano-Reina, E., \& Álvarez-Ude, F. (2019). Disability in instrumental activities of daily living in hemodialysis patients: Influence on quality of life related to health. Nefrologia, 39(5), 531-538. https://doi.org/10.1016/j.nefroe.2019.10.007

Chen, W. Y., Jang, Y., Wang, J. Der, Huang, W. N., Chang, C. C., Mao, H. F., \& Wang, Y. H. (2011). Wheelchair-related accidents: Relationship with wheelchair-using behavior in active community wheelchair users. Archives of Physical Medicine and Rehabilitation, 92(6), 892898. https://doi.org/10.1016/j.apmr.2011.01.008

Gayol-Mérida, D. A., Pérez-Zavala, R., Reyes-Aguilar, P., \& Pineda, C. (2014). Diseño y evaluación de asientos preventivos de úlceras por presión para lesionados medulares. Revista de Investigacion Clínica, 66, 61-69. https://www.medigraphic.com/cgibin/new/resumen.cgi?IDARTICULO $=54905$

Hartigan, I. (2007). A comparative review of the Katz ADL and the Barthel Index in assessing the activities of daily living of older people. International Journal of Older People Nursing, 2(3), 204-212. https://doi.org/10.1111/j.1748-3743.2007.00074.x

Hilgenkamp, T. I. M., van Wijck, R., \& Evenhuis, H. M. (2011). (Instrumental) activities of daily living in older adults with intellectual disabilities. Research in Developmental Disabilities, 32(5), 1977-1987. https://doi.org/10.1016/j.ridd.2011.04.00

INEGI (2018). Encuesta nacional de la dinámica demográfica (ENADID). 
https://www.inegi.org.mx/programas/enadid/2018/

Instituto Nacional de Salud Pública (2013). Encuesta nacional sobre percepcion de la discapacidad en población mexicana. https://encuestas.insp.mx/enpdis/descargas/ENPDis19sept FINAL.pdf

Johnson, N., Barion, A., Rademaker, A., Rehkemper, G., \& Weintraub, S. (2004). The activities of daily living questionnaire: A validation study in patients with dementia. Alzheimer Disease and Associated Disorders, 18(4), 223-230. https://pubmed.ncbi.nlm.nih.gov/15592135/

Lysack, C. L., Zafonte, C. A., Neufeld, S. W., \& Dijkers, M. P. J. M. (2001). Self-care independence after spinal cord injury: Patient and therapist expectations and real life performance. Journal of Spinal Cord Medicine, 24(4), 257-265. https://doi.org/10.1080/10790268.2001.11753583

Millán-Calenti, J. C., Tubío, J., Pita-Fernández, S., González-Abraldes, I., Lorenzo, T., FernándezArruty, T., \& Maseda, A. (2010). Prevalence of functional disability in activities of daily living (ADL), instrumental activities of daily living (IADL) and associated factors, as predictors of morbidity and mortality. Archives of Gerontology and Geriatrics, 50(3), 306-310. https://doi.org/10.1016/j.archger.2009.04.017

Mlinac, M. E., \& Feng, M. C. (2016). Assessment of activities of daily living, self-care, and independence. Archives of Clinical Neuropsychology, 31(6), 506-516. https://doi.org/10.1093/arclin/acw049

Neumann-Collyer, V. E., Hernandez-Perez, K. A., \& Gentoso-Pohl-Montt, P. (2018). Diseño de un instrumento de evaluación de desempeño en actividades de la vida diaria. Revista de Salud Pública, 20(5), 554-559. https://doi.org/10.15446/rsap.v20n5.70909

Norman, D. A. (2005). EL diseño emocional. Paidós.

Organización Mundial de la Salud (2001). Clasificación internacional del funcionamiento, de la discapacidad y de la salud. OMS. https://aspace.org/assets/uploads/publicaciones/e74e4cif 2001.pdf

Organización de las Naciones Unidas (2014). Convención sobre los derechos de las personas con discapacidad y protocolo facultativo. Revista Ciencias de la Salud, 6(23), 115. https://doi.org/10.1017/CBO9781107415324.004

Persad, U., Langdon, P., \& Clarkson, J. (2007). Characterising user capabilities to support inclusive design evaluation. Universal Access in the Information Society, 6(2), 119-135. https://doi.org/10.1007/s10209-007-0083-y

Ran, L., Jiang, X., Li, B., Kong, H., Du, M., Wang, X., Yu, H., \& Liu, Q. (2017). Association among activities of daily living, instrumental activities of daily living and health-related quality of life in elderly Yi ethnic minority. BMC Geriatrics, 17(1), 1-7. https://doi.org/10.1186/s12877017-0455-y

Romero-Ayuso, D. (2007). Actividades de la vida diaria. Anales de Psicología, 23(2), 264-271.

Spector, W. D., \& Fleishman, J. A. (1998). Combining activities of daily living with instrumental activities of daily living to measure functional disability. Journals of Gerontology - Series B Psychological Sciences and Social Sciences, 53(1), 46-56. https://doi.org/10.1093/geronb/53B.1.S46 
Sun, X., Lucas, H., Meng, Q., \& Zhang, Y. (2011). Associations between living arrangements and health-related quality of life of urban elderly people: A study from China. Quality of Life Research, 20(3), 359-369. https://doi.org/10.1007/s11136-010-9752-z

Van Rensbergen, G., \& Pacolet, J. (2012). Instrumental activities of daily living (I-ADL) trigger an urgent request for nursing home admission. Archives of Public Health, 70(1), 1-19. https://doi.org/10.1186/0778-7367-70-2

Visagie, S., Mlambo, T., Van der Veen, J., Nhunzvi, C., Tigere, D., \& Scheffler, E. (2015). Is any wheelchair better than no wheelchair? A Zimbabwean perspective. African Journal of Disability, 4(1), 1-10. https://doi.org/10.4102/ajod.v4i1.201 